\title{
Facial Skin Coloration Affects Perceived Health of Human Faces
}

\author{
Ian D. Stephen • Miriam J. Law Smith • \\ Michael R. Stirrat • David I. Perrett
}

Received: 31 March 2009 /Accepted: 28 September 2009 /

Published online: 26 October 2009

(C) The Author(s) 2009. This article is published with open access at Springerlink.com

\begin{abstract}
Numerous researchers have examined the effects of skin condition, including texture and color, on the perception of health, age, and attractiveness in human faces. They have focused on facial color distribution, homogeneity of pigmentation, or skin quality. We here investigate the role of overall skin color in determining perceptions of health from faces by allowing participants to manipulate the skin portions of color-calibrated Caucasian face photographs along CIELab color axes. To enhance healthy appearance, participants increased skin redness $\left(a^{*}\right)$, providing additional support for previous findings that skin blood color enhances the healthy appearance of faces. Participants also increased skin yellowness $\left(b^{*}\right)$ and lightness $\left(L^{*}\right)$, suggesting a role for high carotenoid and low melanin coloration in the healthy appearance of faces. The color preferences described here resemble the red and yellow color cues to health displayed by many species of nonhuman animals.
\end{abstract}

Keywords beauty $\cdot$ diet $\cdot$ flushing $\cdot$ hemoglobin $\cdot$ UV protection

\section{Introduction}

The face plays a significant role in human interactions, including mate choice (Perrett et al. 1998). Numerous researchers have examined aspects of human faces

I. D. Stephen $(\bowtie) \cdot$ M. R. Stirrat $\cdot$ D. I. Perrett

Perception Lab, School of Psychology, University of St. Andrews, St. Andrews Fife KY16 9JP, Scotland

e-mail: ian@perceptionlab.com

I. D. Stephen

Department of Experimental Psychology, University of Bristol, Bristol BS8 1TU, UK

M. J. Law Smith

Department of Neuropsychiatric Genetics and Psychiatry,

Trinity Centre for Health Sciences, Trinity College Dublin, Dublin 8, Ireland 
that affect perceptions of health, attractiveness, and age, usually focusing on facial shape (Perrett et al. 1999; Rhodes et al. 1999). However, more recently, several studies have focused on the contributions of skin color and texture to apparent facial health and attractiveness (Fink et al. 2001, 2006; Jones et al. 2004; Matts et al. 2007; Stephen et al. 2009). Here, we expand this approach by examining the effects of overall skin color on the appearance of health in human faces while controlling experimentally for texture and shape effects.

Previous researchers have shown that the homogeneity and distribution of skin color across the face contributes to perceptions of health, age, and attractiveness of human faces (Fink et al. 2001, 2006; Matts et al. 2007). Fink et al. (2001) found that ratings of facial attractiveness correlated with measures of skin color homogeneity, such that more homogeneous skin color distribution was more attractive. More homogeneous chromophore (melanin and hemoglobin) distribution across the skin also correlates positively with ratings of health, attractiveness, and youthfulness (Matts et al. 2007), while the facial color distribution of younger women is perceived as younger, healthier, and more attractive than that of older women (Fink et al. 2006). Further, increased contrast between the luminance of the facial features and the rest of the facial skin has been shown to enhance the attractiveness and femininity of female faces, and to reduce the attractiveness and masculinity of male faces (Russell 2003). However, it is not only color distribution that plays a role in indicating facial health. Jones et al. (2004) found that health ratings of skin patches taken from face photographs correlated with ratings of attractiveness of the whole faces, suggesting that skin color and texture themselves contribute to the attractiveness of faces independently of shape, and when color distribution across the whole face cannot be viewed.

Though these studies clearly indicate a role for facial color, they have all either treated the color and texture of skin together, without assessing color effects that are independent of texture effects, or dealt with color distribution, and none has made use of color-calibration techniques (Hong et al. 2001). Recently, we have begun to investigate the role of overall facial skin color in determining the perception of health from faces via the CIELab color space (Stephen et al. 2009).

The CIELab color system is commonly used in human perceptual work, modeled on the human visual system, and designed to be perceptually uniform, with a change of 1 unit appearing to be of approximately the same magnitude regardless of the dimension of the change (Martinkauppi 2002). Here, we use the color manipulation techniques and experimental paradigm established by Stephen et al. (2009) to investigate the effect of overall skin color on the apparent health of human faces. Single-axis transforms allowed participants to manipulate the skin portions of color-calibrated facial photographs along the 3 CIELab component axes: $L^{*}$ (light-dark), $a^{*}$ (red-green), and $b^{*}$ (yellow-blue), separately to optimize apparent health. CIELab $a^{*}$ values are affected primarily by blood in human skin (Edwards and Duntley 1939), and only single-axis transforms were undertaken in the $a^{*}$ dimension. The $b^{*}$ component of skin color is substantially influenced by 2 pigments, carotenoids and melanin, but these pigments have different influences on the $L^{*}$ component (Edwards and Duntley 1939; Stamatas et al. 2004), so for $L^{*}$ 
and $b^{*}$ we undertook both single-axis and 2-dimensional trials, in which we allowed participants to manipulate faces along $L^{*}$ and $b^{*}$ axes simultaneously. To investigate contrast in our study of color images, we investigated the difference between color changes applied when lip color remained constant or was manipulated along with the facial skin.

Increased skin vascularization increases skin $a^{*}$ and is associated with increased physical fitness in humans (Johnson 1998) and increased estrogen levels in women (Charkoudian et al. 1999; Thornton 2002), but is impaired in patients with type 2 diabetes (Charkoudian 2003) and hypertension (Panza et al. 1990). Increased blood oxygenation, associated with increased aerobic fitness (Armstrong and Welsman 2001), increases blood redness. Skin blood deoxygenation is associated with cardiac and respiratory illness, and causes a dull, blue tint to the skin (Ponsonby et al. 1997). Previous perceptual work has shown that increased skin blood perfusion and oxygenation coloration enhance the healthy appearance of human faces (Stephen et al. 2009). Similar phenomena appear to exist in nonhuman primate species, with redness reflecting aspects of underlying physiology. Increased facial skin redness, due to increased skin blood perfusion, has been linked to increased testosterone in male rhesus macaques (Rhodes et al. 1997), and increased estrogen levels and fertility have been linked with increased redness of anogenital skin in female rhesus macaques (Czaja et al. 1977; Dixson 1983). Both of the reddened areas attract preferential visual attention from the opposite sex (Waitt et al. 2003, 2006). Facial redness is also associated with higher testosterone levels and dominance rank in male mandrills (Setchell and Dixson 2001) and reproductive status in female mandrills (Setchell et al. 2006); female mandrills prefer to mate with brightly colored males (Setchell 2005). Facial redness in male mandrills may therefore be an honest signal, demonstrating the bearer's ability to bear the burden of the immune suppression caused by increased testosterone levels (Folstad and Karter 1992; cf. Setchell et al. 2009). We predict that increased skin redness will enhance the healthy appearance of human faces, due to its association with increased blood perfusion and oxygenation.

CIELab $b^{*}$ values are affected primarily by melanin and carotenoids in human skin (Edwards and Duntley 1939; Stamatas et al. 2004). Carotenoids are obtained from consumption of fruits and vegetables (Alaluf et al. 2002), and dietary supplementation causes skin yellowing (Stahl et al. 1998). Carotenoid supplementation is associated with improved development of the immune system in human children (Alexander et al. 1985), whereas individuals infected with HIV and malaria have reduced carotenoid levels (Friis et al. 2001). Carotenoid levels may also affect spermatogenesis in boars (Chew 1993), and women who failed to conceive during in vitro fertilization had unusually fluctuating carotenoid levels in their follicular fluid (Schweigert et al. 2003). Brightly colored carotenoid-based ornaments are displayed by many bird and fish species, the size and brightness of which reflect aspects of health and condition. Male greenfinches with brighter yellow breast feathers showed stronger humoral immune responses to a novel antigen (Saks et al. 2003). Male and female yellow-eyed penguins with more saturated yellow eye ornamentation produced more offspring per season (Massaro et al. 2003). Researchers have demonstrated mate choice based on the brightness of carotenoid ornaments in greenfinches (Eley 1991), yellow-eyed penguins 
(Massaro et al. 2003), and goldfinches (MacDougall and Montgomerie 2003). We predict that participants will increase skin $b^{*}$ in both single-axis and twodimensional trials, indicating a role for carotenoids or melanin in enhancing the appearance of health in faces.

CIELab $L^{*}$ values are affected primarily by melanin, increased levels of which increase skin $b^{*}$ values and decrease skin $L^{*}$ values (Stamatas et al. 2004). Melanin may have health costs, with the UV-filtering effects impairing vitamin D synthesis, potentially leading to rickets and osteomalacia (Jablonski and Chaplin 2000; Murray 1934; $c f$. Robins 1991). Health benefits of melanin include providing protection from damaging UV radiation, reducing the incidence of skin cancer and sunburn (Robins 1991), and reducing the photolysis of folate, a deficiency of which during pregnancy places the fetus at risk of neural tube defects (Omaye 1993). Melanin plays a role in immune defense in humans (Burkhart and Burkhart 2005), as in insects (Boman and Hultmark 1987). Researchers have long noted that men have darker skin than women (Edwards and Duntley 1939; Hulse 1967; van den Berghe and Frost 1986), and have suggested that light skin is considered more attractive, particularly in female faces where it may be taken as a sign of femininity, youth, and fecundity (Aoki 2002; Jones 2000; van den Berghe and Frost 1986; Wagatsuma 1968) or high social class in both sexes (Hulse 1967; Jones 2000). Several researchers have addressed the perception of human skin lightness, though they have provided conflicting results and used unreliable methodologies, often relying on questionnaires (Feinmann and Gill 1978), uncalibrated stimuli (Frost 1994), or correlational studies (Jones 2000). In our study, in both the single-axis and two-dimensional trials, a role for melanin pigmentation in enhancing the apparent health of faces would be indicated if participants increase $b^{*}$ values and decrease $L^{*}$ values, indicating a preference for a tan color. If participants increase $b^{*}$ coloration but do not decrease $L^{*}$ values, a role for carotenoids will be indicated. Because carotenoids are associated with health signaling in many species, whereas melanin is associated with both health costs and benefits, we predict that participants will not prefer to reduce $L^{*}$ values in faces to optimize healthy appearance.

Previous studies of skin color have suggested a role for sexual dimorphism in color preferences, with female skin being lighter in females in many populations, and lightness appearing more attractive in females (van den Berghe and Frost 1986). We predict that participants will prefer to enhance sexual dimorphism in faces by increasing $L^{*}$ in female faces more than in male faces, and increasing $a^{*}$ and $b^{*}$ more in male than in female faces.

Following Russell's (2003) findings on facial contrast, we predict that manipulations that increase the contrast between the features and the skin (the lips constant conditions) will be enhanced more in female faces than in male faces.

\section{Materials and Methods}

This work was approved by the University of St. Andrews Ethics Committee. All participants gave prior, informed consent in writing. 


\section{Photography}

We photographed 51 Caucasian participants (21 male, 30 female; ages 18-22) without skin makeup and with neutral expressions, in a booth painted Munsell N5 gray, illuminated with 3 Verivide F20 T12/D65 daylight simulation bulbs in highfrequency fixtures (Verivide, UK), to reduce the effects of flicker. The booth was located in a room with no other lighting. We placed a Munsell N5 painted board over the shoulders and included a GretagMacbeth Mini ColorChecker color chart in the frame. We color-corrected images using a least-squares transform, from an 11expression polynomial expansion (Hong et al. 2001) of camera RGB values for 24 ColorChecker patches to the manufacturer-specified CIELab values of the same patches. This achieved a mean color error $(\Delta E)$ of 2.44 between the 24 manufacturer stated color values and the color values obtained from the corrected images. ( $\Delta E$ is the Euclidean distance between 2 color points in CIELab space, and is the standard method used for quoting color differences in CIELab color space.)

We used Matlab to calculate mean CIELab values across skin pixels for each face image (defining initial CIELab face color).

\section{Image Manipulation}

We used Matlab to produce masks with even coloration representing the skin areas of faces (with a Gaussian blur at the edges), 1 representing average face color +8 units of $a^{*}$ (increased redness) and 1 with average face color -8 units of $a^{*}$ (decreased redness). The masks were warped to the shape of the skin portions of each face image $(n=51)$ in turn. We manipulated each face by the difference in color between each of the pairs of masks (Burt and Perrett 1995). This produced a single manipulation composed of a series of 13 frames (numbered 0-12), in which frame 0 had skin redness reduced by 16 units of $a^{*}$, increasing incrementally so that frame 6 was the original image and frame 12 had skin redness increased by 16 units of $a^{*}$ (Fig. 1a).

For the single-axis transforms, we repeated this procedure for each of the 51 faces, in each of 3 color dimensions ( $L^{*}, a^{*}$, and $b^{*}$; Figs. 2a and $3 a$ ) and in each of 2 lip conditions (lip color changes with the rest of the facial skin, and lip color remains constant as the facial skin color changes), giving a total of 306 (51 faces $\times 3$ color dimensions $\times 2$ lip conditions) manipulations to be presented to participants. Each of these manipulations comprised a series of 13 frames. Each of the 306 trials allowed a participant to cycle through the 13 frames making up the manipulation by moving the mouse horizontally across the screen, in order to select the frame that appears the healthiest.

2D transforms allowed the participants to manipulate the 51 faces simultaneously along $L^{*}$ and $b^{*}$ color axes to optimize healthy appearance. To generate these 2D transforms, we transformed each of the 51 faces along the $L^{*}$ axis (13 frames), and then along the $b^{*}$ axis (13 frames), meaning that this manipulation was made up of 169 frames $(13 \times 13$; Fig. $4 a)$. This can be envisioned as a grid, 13 cells high, e.g., $L^{*} 0$ to $L^{*} 12$, by 13 cells across, e.g., $b^{*} 0$ to $b^{*} 12$ ). When the mouse pointer passes over each cell, the corresponding frame of the manipulation is displayed. Therefore, for each of the 51 trials, moving the mouse horizontally would change the facial skin 

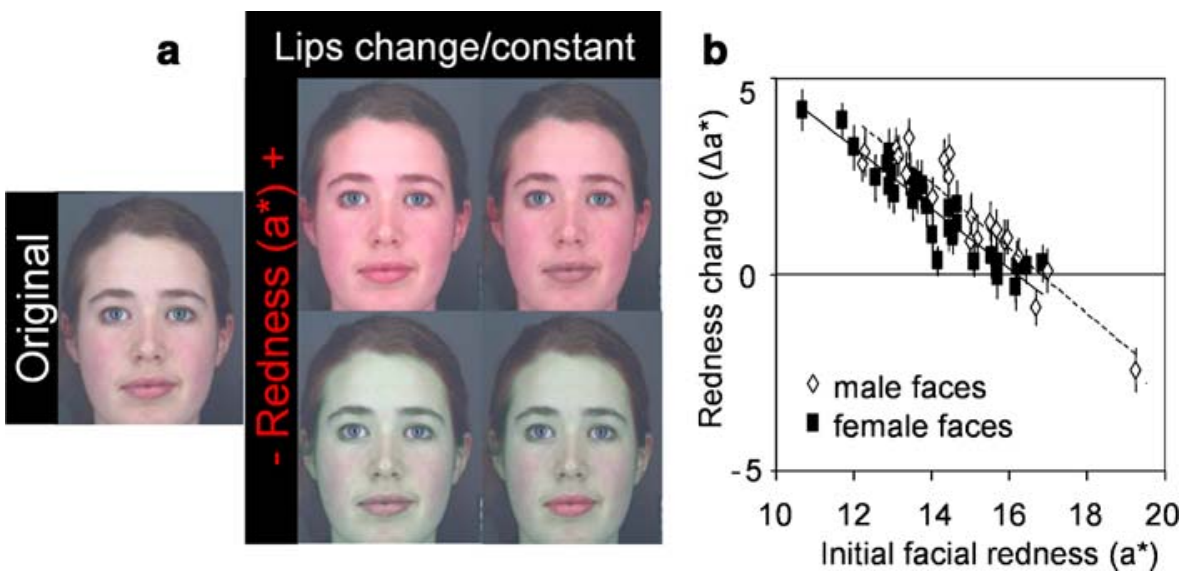

Fig. 1 (a) Original facial image and endpoint images of the redness transform showing images with increased (top) and decreased (bottom) redness ( \pm 16 units of $\left.a^{*}\right)$ in lips changing (left) and constant (right) conditions. (b) For the range of color-calibrated face colors presented, participants increased redness $\left(a^{*}\right)$ to optimize healthy appearance, particularly in male faces. Solid rectangles and regression line $\left(R^{2}=0.87\right)$ represent female faces. Hollow rhombi and dashed regression line $\left(R^{2}=0.86\right)$ represent male faces.

in 1 color dimension - randomly assigned to either $L^{*}$ or $b^{*}$-and moving the mouse vertically would change the skin in the other color dimension, allowing both $L^{*}$ and $b^{*}$ color dimensions to be changed simultaneously. For the 2D transforms, we manipulated lip color along with the facial skin.

For all transforms, hair, eyes, clothing, and background remained constant.

\section{Experimentation}

We recruited 32 participants (12 male, 20 female, ages 19-26) for manipulations along single-color axes and 22 participants (12 male, 10 female, ages $18-25)$ for 2D trials. All participants self-identified as Caucasian.

For the single-axis transforms, we presented participants with the 306 trials, 1 face at a time in random order on a CRT monitor (color-calibrated using a ColorVision Spyder 2Pro to mean $\Delta E$ of 2.32 for a range of skin tones reflecting faces of various ethnicities). Each participant saw each of the 51 face identities only once for each type of trial. For all trials, the participants were given the instruction: make the face as healthy as possible.

\section{Statistical Methods}

We performed statistical analyses via SPSS version 16. We calculated mean color changes applied to each face (by face dataset). We used 1-sample $t$-tests $\left(\mathrm{H}_{0}\right.$ : no color change) to evaluate the overall color changes. We used General Linear Mixed Models to test for the effects of initial face color, face and participant sex, and lip condition, on color change applied (dependent variable = color change; fixed factors = face sex, participant sex, lip condition; random factor $=$ participant ID; covariates = initial face color: $L^{*}, a^{*}$, and $b^{*}$ ). We included all main effects in the model. We 

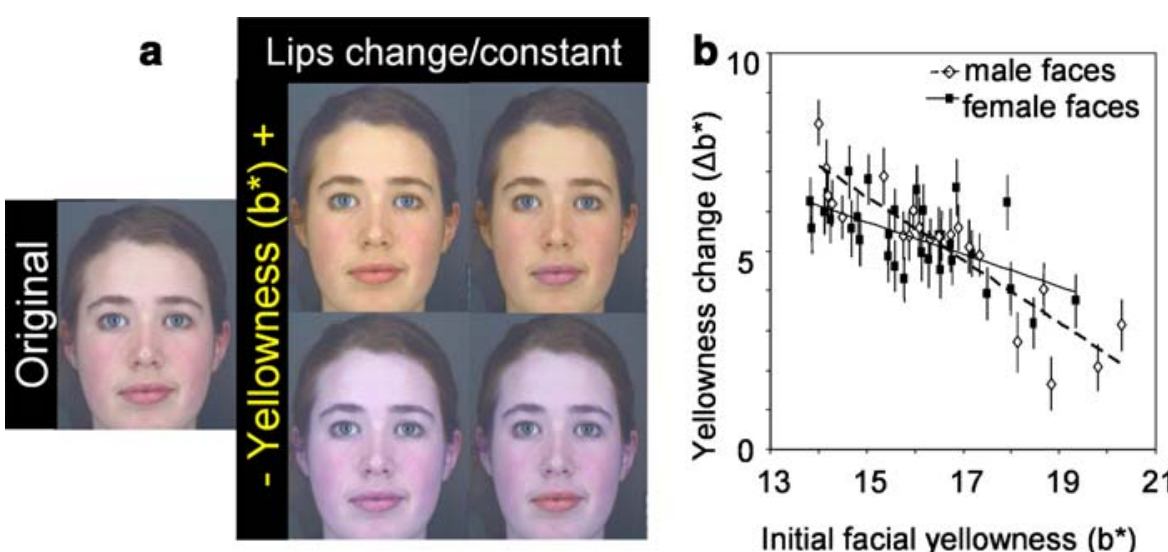

Initial facial yellowness $\left(b^{\star}\right)$

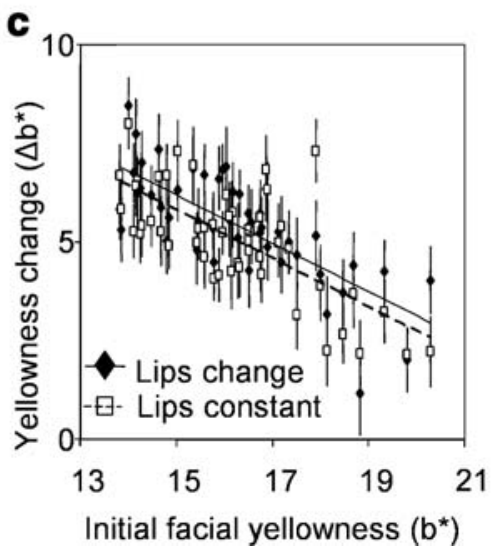

Fig. 2 (a) Original facial image and endpoint images of the yellowness transform showing images with increased (top) and decreased (bottom) yellowness ( \pm 16 units of $\left.b^{*}\right)$ in lips changing (left) and constant (right) conditions. (b) For the range of color-calibrated face colors presented, participants increase yellowness $\left(b^{*}\right)$ to optimize healthy appearance, particularly in male faces. Solid rectangles and regression line $\left(R^{2}=0.22\right)$ represent female faces. Hollow rhombi and dashed regression line $\left(R^{2}=0.80\right)$ represent male faces. (c) Participants increase yellowness more when the lips change color along with the rest of the facial skin than when the lips remain constant. Solid rhombi and regression line $\left(R^{2}=0.55\right)$ represent faces in the lips changing condition. Hollow rectangles and dashed regression line $\left(R^{2}=0.50\right)$ represent faces in the lips constant condition.

nested participant ID within participant sex. The interactions between participant sex and face sex and between face sex and lip condition were included in the model.

\section{Results}

For the range of color-calibrated face colors presented here, participants increased $a^{*}$ (mean redness change \pm SE $\Delta a^{*}=1.62 \pm 0.19 ; t_{50}=8.71 ; p<0.001 ;$ Fig. $1 b$ ) to optimize healthy appearance. Statistical results from the General Linear Mixed Modeling analyses are summarized in Table I. The amount of color change applied to optimize healthy appearance was affected by the initial color of the face, 


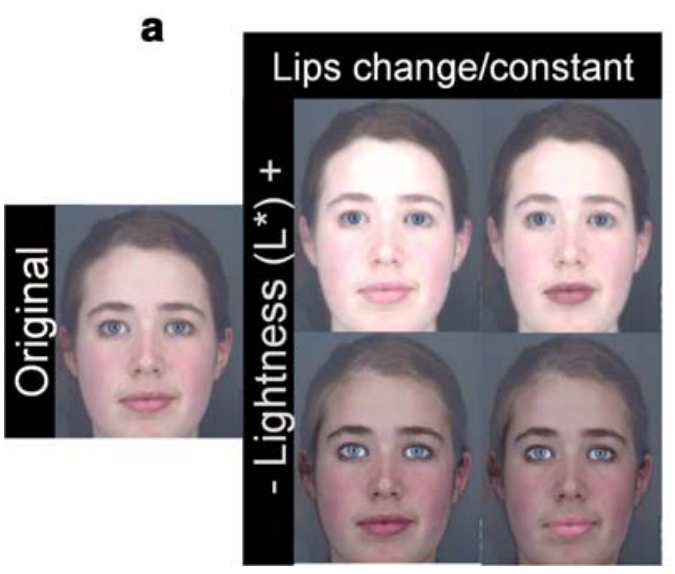

\section{b}

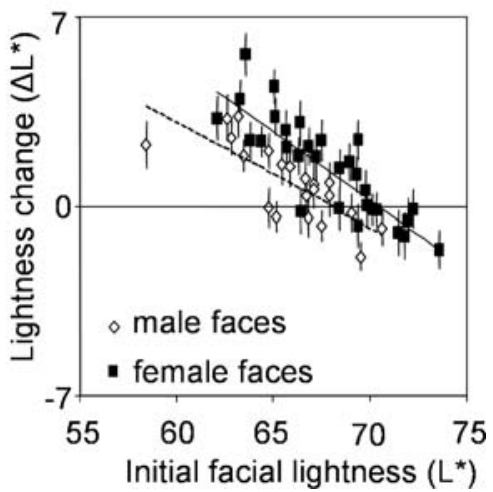

Fig. 3 (a) Original facial image and endpoint images of the lightness transform showing images with increased (top) and decreased (bottom) lightness ( \pm 16 units of $\left.L^{*}\right)$ in lips changing (left) and constant (right) conditions. (b) For the range of color-calibrated face colors presented, participants increased lightness $\left(L^{*}\right)$ to optimize healthy appearance, particularly in female faces. Solid rectangles and regression line $\left(R^{2}=0.73\right)$ represent female faces. Hollow rhombi and dashed regression line $\left(R^{2}=0.59\right)$ represent male faces.

particularly by the dimension being manipulated. Participants increased $a^{*}$ more in faces initially low in a* (Fig. 1b) and, to a lesser extent, in faces high in $b^{*}$.

Participants increased $b^{*}\left(\Delta b^{*}=5.25 \pm 0.18 ; t_{50}=29.64 ; p<0.001 ;\right.$ Fig. $\left.2 b\right)$ to optimize healthy appearance in the single-axis trials. Participants increased $b^{*}$ more in faces initially low in $b^{*}$ (Fig. 2b) and in faces initially high in $L^{*}$ and $a^{*}$.

Participants increased $L^{*}\left(\Delta L^{*}=1.21 \pm 0.23 ; t_{50}=5.20 ; p<0.001 ;\right.$ Fig. $\left.3 b\right)$ to optimize healthy appearance. Participants increased $L^{*}$ more in faces initially low in $L^{*}$ (Fig. 3b) and, to a lesser extent, also in faces low in $a^{*}$.

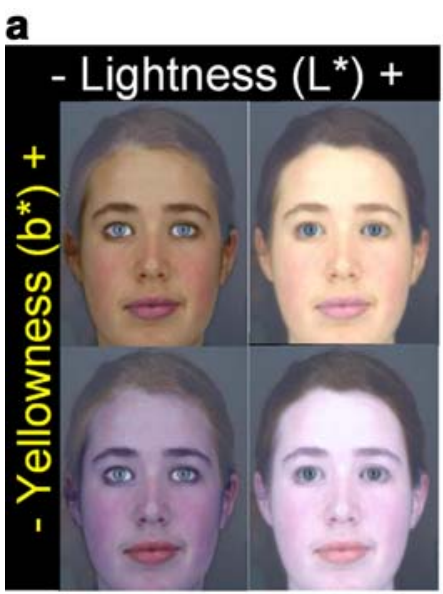

b

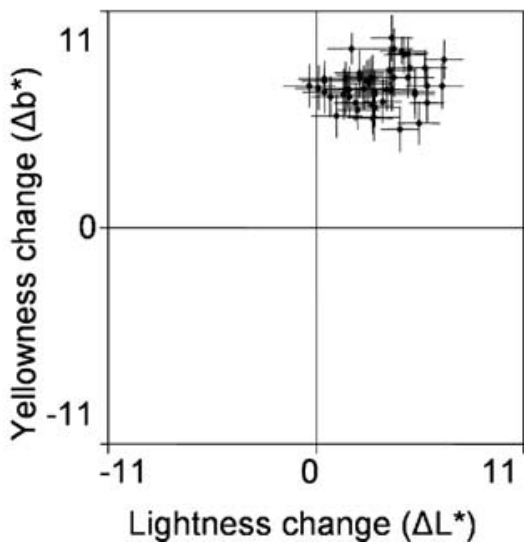

Fig. 4 (a) Endpoints of the two-dimensional $L^{*}$ vs. $b^{*}$ transform. (b) For the range of color-calibrated face colors presented, participants increase both yellowness and lightness to optimize healthy appearance. 
Table I Summarized statistics from the General Linear Mixed Modeling analysis

\begin{tabular}{llll}
\hline & \multicolumn{2}{l}{ Dimension manipulated } & \\
\cline { 2 - 4 } & $L^{*}$ & $a^{*}$ & $b^{*}$ \\
\hline Lips & $F_{1,3326}=0.01 ; p=0.939$ & $F_{1,3326}=1.12 ; p=0.290$ & $F_{1,3326}=9.02 ; p=0.003$ \\
Lips * face sex & $F_{1,3326}=0.03 ; p=0.855$ & $F_{1,3326}=0.81 ; p=0.370$ & $F_{1,3326}=0.70 ; p=0.404$ \\
$L^{*}$ & $F_{1,3326}=165.41 ; p<0.001$ & $F_{1,3326}=0.12 ; p=0.730$ & $F_{1,3326}=77.47 ; p<0.001$ \\
$a^{*}$ & $F_{1,3326}=4.64 ; p=0.031$ & $F_{1,3326}=484.32 ; p<0.001$ & $F_{1,3326}=5.05 ; p=0.025$ \\
$b^{*}$ & $F_{1,3326}=0.31 ; p=0.859$ & $F_{1,3326}=4.31 ; p=0.038$ & $F_{1,3326}=276.19 ; p<0.001$ \\
Participant sex & $F_{1,31.06}=3.66 ; p=0.065$ & $F_{1,31.05}=0.67 ; p=0.420$ & $F_{1,31.05}=1.13 ; p=0.297$ \\
Face sex & $F_{1,3326}=79.42 ; p<0.001$ & $F_{1,3326}=19.00 ; p<0.001$ & $F_{1,3326}=25.84 ; p<0.001$ \\
Participant sex * face sex & $F_{1,3326}=0.10 ; p=0.747$ & $F_{1,3326}=0.35 ; p=0.556$ & $F_{1,3326}=1.26 ; p=0.262$ \\
Participant sex & $F_{31,3326}=34.08 ; p<0.001$ & $F_{31,3326}=43.76 ; p<0.001$ & $F_{31,3326}=43.62 ; p<0.001$ \\
(participant number) & & & \\
\hline
\end{tabular}

In the $2 \mathrm{D}$ trials, participants increased $b^{*}\left(\Delta b^{*}=0.463 \pm 0.01 ; t_{50}=49.940\right.$; $p<0.001)$ and $L^{*}\left(\Delta L^{*}=0.181 \pm 0.12 ; t_{50}=10.629 ; \mathrm{p}<0.001\right)$ to optimize healthy appearance (Fig. 4b).

Participants added more $a^{*}$ (Fig. 1b) and $b^{*}$ (Fig. 2b) to male faces than to female faces and more $L^{*}$ to female faces than to male faces (Fig. 3b). In our sample, female faces are originally lighter than male faces $\left(t_{49}=2.342 ; p=0.023\right)$.

The lips transform condition did not affect the color change applied in the $L^{*}$ or $a^{*}$ transforms. Participants increased $b^{*}$ in faces more when lips changed along with the face than when they remained constant (Fig. 2c), though they increased $b^{*}$ in both conditions. There was no interaction effect of lip condition with sex of face in any of the 3 dimensions.

\section{Discussion}

Our study reveals a role of overall skin color in the appearance of health in human faces, separate from information provided by skin texture or color distribution. We have shown that, for a sample of 51 color-calibrated Caucasian face photographs, increased skin $a^{*}, b^{*}$, and $L^{*}$ enhance the perceived health of human faces. Participants increased color more in faces initially low in that dimension, suggesting that extremes of skin coloration outside the natural range do not appear healthy, possibly because of preferences for face color within the normal range (Langlois and Roggman 1990).

Stephen et al. (2009) found that the physiologically relevant cues of increased skin blood perfusion and oxygenation color enhance the healthy appearance of faces. The current study takes a more perceptual stance, demonstrating that pure color changes along dimensions modeled on the human visual system (Martinkauppi 2002) affect the apparent health of human faces. We found that skin redness enhanced the perceived health of faces, possibly because of its association with skin 
blood perfusion and oxygenation (Edwards and Duntley 1939; Stephen et al. 2009). This relationship shows similarities to color signals displayed in nonhuman primates, particularly Old World monkeys (Dixson 1998). Increased redness due to increased blood perfusion is associated with aspects of health such as hormonal status (Czaja et al. 1977; Dixson 1983; Rhodes et al. 1997; Setchell and Dixson 2001) and reproductive status (Rhodes et al. 1997; Setchell et al. 2006) in this group. Redness in nonhuman primates is also associated with social factors such as dominance rank (Setchell and Dixson 2001; Setchell and Wickings 2005) and is preferred by the opposite sex (Setchell 2005; Waitt et al. 2003, 2006). Investigation of the role of facial redness in the appearance of human dominance and attractiveness is therefore warranted.

It is a popular view that suntanning enhances the healthy and attractive appearance of Caucasian faces. The role of melanin in the perception of faces is usually considered in terms of skin darkening (reduced $L^{*}$; Frost 1994; van den Berghe and Frost 1986). Our finding that participants choose to increase $b^{*}$ and increase $L^{*}$ in both single-axis and 2D trials suggests that any benefit to health appearance from tanning is due to the associated increase in skin $b^{*}$ (Stamatas et al. 2004). We suggest that the healthy appearance of skin $b^{*}$ may be attributable to dietary carotenoid deposition in the skin. This suggests that carotenoids, which are involved in health signaling (Massaro et al. 2003; Saks et al. 2003) and sexual selection (Eley 1991; MacDougall and Montgomerie 2003; Massaro et al. 2003) in many species of birds and fish, may also affect the appearance of health in humans. To clarify the relative roles of these pigments in facial health, a study investigating the role of skin melanin and carotenoid coloration on the apparent health of human faces would be a valuable next step.

Participants exaggerated existing sexual dimorphism in skin color by lightening female faces more than male faces and reddening and yellowing male faces more than female faces. This is congruent with the finding that men are browner, ruddier (Edwards and Duntley 1939; Frost 1994), and darker (van den Berghe and Frost 1986) than women in many populations and may be attributed to a preference for faces that conform to sexually dimorphic patterns (Perrett et al. 1998; Rhodes 2006).

While there were no effects of lip condition in $a^{*}$ or $L^{*}$ trials, participants increased skin $b^{*}$ more when the lip color changed along with the rest of the facial skin. When the lips remained constant as the rest of the facial skin was increased in $b^{*}$, the lips appeared blue (Fig. 3a), a symptom of cyanosis (a lack of blood oxygenation that is associated with cardiac and respiratory illnesses; Ponsonby et al. 1997). We did not find any difference in the effect of lip condition between the 2 sexes in any of the 3 color dimensions. These findings differ from those of Russell (2003), who found that increased contrast between the features and the facial skin enhanced femininity and attractiveness in female faces but reduced masculinity and attractiveness in male faces. One of 3 explanations may account for these results: 1) Russell's use of black-and-white photographs may have eliminated important color information from his images. 2) The contrast between the features and the facial skin may be important for attractiveness and sexual dimorphism perception, but not for health perception. 3) The effects found by Russell (2003) may be attributable to the contrast between the eyes and the skin instead of the lips and the skin. A more detailed study of these issues is therefore warranted. 


\section{Summary}

We have established a role for overall skin color, independent of skin texture or color distribution, in the appearance of health in human faces. The enhancement of healthy appearance caused by increased skin redness provides additional support for a role for blood coloration in health perception (Stephen et al. 2009). The role of skin yellowness and lightness suggests that carotenoid pigmentation may also play a role in health perception. Together, the results suggest that colors that are associated with health signaling and mate choice in a number of animal species also play a role in the perception of health in human faces.

Acknowledgments We thank P. Hibbard, A. Hurlburt, Y. Ling, I. Penton-Voak, and T. Troscianko for color calibration advice; M. Anderson, R. Byrne, R. Caldara, P. Ferretti, T. Fitch, P. Hancock, B. Jones, M. Johnston, D. Johnston, J. Lawson, J. Seddon, R. Sprengelmeyer, M. Tovée, D. Vishnawath, J. Higham, J. Setchell, and 2 anonymous reviewers for comments; P. Wilcox for booth fabrication; J. Schindelin for additional computer programs; L. Ferrier for organizing participants; and A. Perrett for proofreading. This work was supported by the BBSRC, Unilever Research, and the ESRC.

Open Access This article is distributed under the terms of the Creative Commons Attribution Noncommercial License which permits any noncommercial use, distribution, and reproduction in any medium, provided the original author(s) and source are credited.

\section{References}

Alaluf, S., Heinrich, U., Stahl, W., Tronnier, H., \& Wiseman, S. (2002). Dietary carotenoids contribute to normal human skin color and UV photosensitivity. Journal of Nutrition, 132, 399-403.

Alexander, M., Newmark, H., \& Miller, R. G. (1985). Oral $\beta$-carotene can increase the number of OKT4+ cells in human blood. Immunology Letters, 9, 221-224.

Aoki, K. (2002). Sexual selection as a cause of human skin color variation: Darwin's hypothesis revisited. Annals of Human Biology, 29, 589-608.

Armstrong, N., \& Welsman, J. R. (2001). Peak oxygen uptake in relation to growth and maturation in 11to 17-year-old humans. European Journal of Applied Physiology, 85, 546-551.

Boman, H. G., \& Hultmark, D. (1987). Cell-free immunity in insects. Annual Review of Microbiology, 41, $103-126$.

Burkhart, C. G., \& Burkhart, C. N. (2005). The mole theory: Primary function of melanocytes and melanin may be antimicrobial defense and immunomodulation (not solar protection). International Journal of Dermatology, 44, 340-342. doi:10.1111/j.1365-4632.2004.02556.x.

Burt, D. M., \& Perrett, D. I. (1995). Perception of age in adult Caucasian male faces: Computer graphic manipulation of shape and color information. Proceedings of the Royal Society of London B, 259, 137-143.

Charkoudian, N. (2003). Skin blood flow in adult human thermoregulation: How it works, when it does not, and why. Mayo Clinic Proceedings, 78, 603-612.

Charkoudian, N., Stephens, D. P., Pirkle, K. C., Kosiba, W. A., \& Johnson, J. M. (1999). Influence of female reproductive hormones on local thermal control of skin blood flow. Journal of Applied Physiology, 87, 1719-1723.

Chew, B. P. (1993). Effects of supplemental $\beta$-carotene and vitamin A on reproduction in swine. Journal of Animal Science, 71, 247-252.

Czaja, J. A., Pobinson, J. A., Eisele, S. G., Scheffler, G., \& Goy, R. W. (1977). Relationship between sexual skin color of female rhesus monkeys and midcycle plasma levels of oestradiol and progesterone. Journal of Reproduction and Fertility, 49, 147-150.

Dixson, A. F. (1983). Observations on the evolution and behavioral significance of "sexual skin" in female primates. Advances in the Study of Behavior, 13, 63-106. 
Dixson, A. F. (1998). Primate sexuality: Comparative studies of the prosimians, monkeys, apes and human beings. Oxford: Oxford University Press.

Edwards, E. A., \& Duntley, S. Q. (1939). The pigments and color of living human skin. American Journal of Anatomy, 65, 1-33.

Eley, C. (1991). Status signaling in the western greenfinch (Carduelis chloris). Ph.D. thesis, University of Sussex, Brighton, U.K.

Feinmann, S., \& Gill, G. W. (1978). Sex differences in physical attractiveness preferences. Journal of Social Psychology, 105, 43-52.

Fink, B., Grammer, K., \& Thornhill, R. (2001). Human (Homo sapiens) facial attractiveness in relation to skin texture and color. Journal of Comparative Psychology, 115, 92-99.

Fink, B., Grammer, K., \& Matts, P. J. (2006). Visible skin color distribution plays a role in the perception of age, attractiveness, and health in female faces. Evolution and Human Behavior, 27, 433-442.

Folstad, I., \& Karter, A. J. (1992). Parasites, bright males and the immunocompetence hypothesis. American Naturalist, 139, 603-622.

Friis, H., Gomo, E., Kastel, P., Ndhlovu, P., Nyazema, N., et al. (2001). HIV and other predictors of serum $\beta$-carotene and retinol in pregnancy: A cross-sectional study in Zimbabwe. American Journal of Clinical Nutrition, 73, 1058-1065.

Frost, P. (1994). Preference for darker faces in photographs at different phases of the menstrual cycle: Preliminary assessment of evidence for a hormonal relationship. Perceptual and Motor Skills, 79, 507-514.

Hong, G., Luo, M. R., \& Rhodes, P. A. (2001). A study of digital camera colorimetric characterization based on polynomial modeling. Color Research \& Application, 26, 76-84.

Hulse, F. S. (1967). Selection for skin color among the Japanese. American Journal of Physical Anthropology, 27, 143-156.

Jablonski, N. G., \& Chaplin, G. (2000). The evolution of human skin color. Journal of Human Evolution, 39, 57-106.

Johnson, J. M. (1998). Physical training and the control of skin blood flow. Medical Science in Sports and Exercise, 30, 382-386.

Jones, D. (2000). Physical attractiveness, race and somatic prejudice in Bahia, Brazil. In L. Cronk, N. Chagnon \& W. Irons (Eds.), Adaptation and human behavior: An anthropological perspective (pp. 133-152). Edison, NJ: Aldine Transaction.

Jones, B. C., Little, A. C., Burt, D. M., \& Perrett, D. I. (2004). When facial attractiveness is only skin deep. Perception, 33, 569-576.

Langlois, J. H., \& Roggman, L. A. (1990). Attractive faces are only average. Psychological Science, 1, 115-121.

MacDougall, A. K., \& Montgomerie, R. (2003). Assortative mating by carotenoid-based plumage color: A quality indicator in American goldfinches, Carduelis tristis. Naturwissenschaften, 90, 464-467.

Martinkauppi, B. (2002). Face color under varying illumination-analysis and applications. Oulu: Oulu University Press.

Massaro, M., Davis, L., \& Darby, J. (2003). Carotenoid-derived ornaments reflect parental quality in male and female yellow-eyed penguins (Megadyptes antipodes). Behavioral Ecology and Sociobiology, 55, $169-175$

Matts, P. J., Fink, B., Grammer, K., \& Burquest, M. (2007). Color homogeneity and visual perception of age, health, and attractiveness of female facial skin. Journal of the American Academy of Dermatology, 57, 977-984.

Murray, F. G. (1934). Pigmentation, sunlight and nutritional disease. American Anthropologist, 36, 438448.

Omaye, D. T. (1993). Nutrient deficiencies and pregnancy outcome. In R. P. Sharma (Ed.), Dietary factors and birth defects (pp. 12-41). San Francisco: Pacific Division AAAS.

Panza, J. A., Quyyimi, A. A., Brush, J. R., \& Epstein, S. E. (1990). Abnormal endothelium-dependent vascular relaxation in patients with essential hypertension. New England Journal of Medicine, 323, 22-27.

Perrett, D. I., Lee, K. J., Penton-Voak, I. S., Rowland, D., Yoshikawa, S., et al. (1998). Effects of sexual dimorphism on facial attractiveness. Nature, 394, 884-887.

Perrett, D. I., Burt, D. M., Penton-Voak, I. S., Lee, K. J., Rowland, D. A., \& Edwards, R. (1999). Symmetry and human facial attractiveness. Evolution and Human Behavior, 20, 295-307.

Ponsonby, A.-L., Dwyer, T., \& Couper, D. (1997). Sleeping position, infant apnea, and cyanosis: a population-based study. Pediatrics, 99, e3. 
Rhodes, G. (2006). The evolutionary psychology of facial beauty. Annual Review of Psychology, 57, 199226.

Rhodes, L., Argersinger, M. E., Gantert, L. T., Friscino, B. H., Hom, G., et al. (1997). Effects of administration of testosterone, dihydrotestosterone, estrogen and fadrozole, an aromatase inhibitor, on sex skin color in intact male rhesus macaques. Journal of Reproduction and Fertility, 111, 51-57.

Rhodes, G., Sumich, A., \& Byatt, G. (1999). Are average facial configurations attractive only because of their symmetry? Psychological Science, 10, 52-58.

Robins, A. H. (1991). Biological perspectives on human pigmentation. Cambridge, UK: Cambridge University Press.

Russell, R. (2003). Sex, beauty and the relative luminance of facial features. Perception, 32, 1093-1107.

Saks, L., Ots, I., \& Horak, P. (2003). Carotenoid-based plumage coloration of male greenfinches reflects health and immunocompetence. Oecologia, 134, 301-307.

Schweigert, F. J., Steinhagen, B., Raila, J., Siemann, A., Peet, D., \& Buscher, U. (2003). Concentrations of carotenoids, retinol and \{alpha\}-tocopherol in plasma and follicular fluid of women undergoing IVF. Human Reproduction, 18, 1259-1264.

Setchell, J. M. (2005). Do female mandrills prefer brightly colored males? International Journal of Primatology, 26, 715-735.

Setchell, J. M., \& Dixson, A. F. (2001). Changes in the secondary sexual adornments of male mandrills (Mandrillus sphinx) are associated with gain and loss of alpha status. Hormones and Behavior, 39, 177-184.

Setchell, J. M., \& Wickings, E. J. (2005). Dominance, status signals and coloration in male mandrills (Mandrillus sphinx). Ethology, 111, 25-50.

Setchell, J. M., Wickings, E. J., \& Knapp, L. A. (2006). Signal content of red facial coloration in female mandrills (Mandrillus sphinx). Proceedings of the Royal Society of London B, 273, 2395-2400.

Setchell, J. M., Charpentier, M. J. E., Abbott, K. M., Wickings, E. J., \& Knapp, L. A. (2009). Is brightest best? Testing the Hamilton-Zuk hypothesis in Mandrills. International Journal of Primatology, 30. doi:10.1007/s10764-009-9371-0.

Stahl, W., Heinrich, U., Jungmann, H., von Laar, J., Schietzel, M., et al. (1998). Increased dermal carotenoid levels assessed by noninvasive reflection spectrophotometry correlate with serum levels in women ingesting Betatene. Journal of Nutrition, 128, 903-907.

Stamatas, G. N., Zmudzka, B. Z., Kollias, N., \& Beer, J. Z. (2004). Non-Invasive measurements of skin pigmentation in situ. Pigment Cell Research, 17, 618-626.

Stephen, I. D., Coetzee, V., Law Smith, M. J., \& Perrett, D. I. (2009). Skin blood perfusion and oxygenation color affect perceived human health. PLoS ONE, 4, e5083.

Thornton, M. J. (2002). The biological actions of estrogens on skin. Experimental Dermatology, 11, 487502 .

van den Berghe, P. L., \& Frost, P. (1986). Skin color preference, sexual dimorphism and sexual selection: A case of gene-culture coevolution? Ethnic and Racial Studies, 9, 87-119.

Wagatsuma, H. (1968). The social perception of skin color in Japan. In J. H. Franklin (Ed.), Color and race. Boston: Houghton Mifflin.

Waitt, C., Little, A. C., Wolfensohn, S., Honess, P., Brown, A. P., et al. (2003). Evidence from rhesus macaques suggests that male coloration plays a role in female primate mate choice. Proceedings of the Royal Society of London B, 270, S144-S146.

Waitt, C., Gerald, M. S., Little, A. C., \& Kraiselburd, E. (2006). Selective attention toward female secondary sexual color in male rhesus macaques. American Journal of Primatology, 68, 738-744. 